\title{
Il welfare aziendale in Italia
}

\author{
Andrea Ciarini*, Silvia Lucciarini**
}

\section{Il contesto: tra welfare e relazioni industriali}

In Italia l'attenzione degli studi sul welfare verso la contrattazione integrativa e le relazioni industriali più in generale è costantemente aumentata negli ultimi anni. Ne è testimone il numero crescente di studi e ricerche sul welfare contrattuale e aziendale (Gori, 2012; Pavolini, Mirabile e Ascoli, 2013; Treu, 2013). Sullo sviluppo di questi istituti, contrattati dentro e fuori le aziende, incidono diversi fattori. Molti di essi sono legati non a un avanzamento delle condizioni entro cui vengono praticate la rappresentanza collettiva e l'offerta di protezione sociale. È anzi in ragione del restringimento del perimetro del welfare pubblico e dell'indebolimento della contrattazione collettiva che welfare e relazioni industriali hanno iniziato a trovare nuove ragioni di integrazione e funzionalità reciproche (Regalia, 2012). Si pensi appunto al crescente sviluppo del welfare contrattato a livello territoriale o di categoria, per ambiti di intervento tradizionali come la previdenza e l'assistenza sanitaria integrativa, ma anche nuovi come i servizi di cura e conciliazione. Si pensi inoltre, ed è quello che in questo lavoro più ci interessa, ai servizi di welfare erogati dalle aziende attraverso accordi contrattati tra le parti o come scelte unilaterali delle imprese o ancora attraverso processi concertativi che vedono coinvolti le imprese, le organizzazioni sindacali e le amministrazioni territoriali.

* Dipartimento di Scienze Sociali ed Economiche, Sapienza Università di Roma.

** Dipartimento di Scienze Sociali ed Economiche, Sapienza Università di Roma.

Questo articolo è frutto di una riflessione comune dei due autori. La stesura dei paragrafi 1 e 4 è da attribuire a Andrea Ciarini. Quella dei paragrafi 2 e 3 a Silvia Lucciarini.

Sociologia del lavoro, n. 139/2015 
Dal punto di vista interno alle aziende la scelta di dotarsi di strumenti di welfare autonomi rispetto a quelli ordinari previsti dal sistema di protezione sociale costituisce una delle modalità attraverso cui incidere sull'aumento della produttività del lavoro, soprattutto del personale più qualificato. A questo scopo anche la legislazione, in special modo il testo unico delle imposte (Mallone, 2013; Treu, 2013), ha costituito un elemento di spinta per il welfare aziendale, rendendo conveniente per le imprese, con sgravi e agevolazioni fiscali, l'offerta di prestazioni integrative rispetto ai semplici aumenti contrattuali.

Per le imprese di più grandi dimensioni la possibilità di operare economie di scala, potendo contare su un numero di addetti elevato e su strutture fisse in grado di ospitare spazi dedicati o servizi integrativi è un fattore di indubbio vantaggio. Vi sono tuttavia criticità non di poco conto in questo senso, considerando il fatto che le grandi imprese non solo sono minoritarie nel sistema produttivo nazionale, ma anche concentrate territorialmente in alcune aree. Viceversa, per i territori a economia diffusa, quelli della piccola e media impresa, un'alternativa su cui si sta sollevando una certa attenzione (Ferrera e Maino, 2013; Mallone, 2013; Treu, 2013) sembra risiedere nella costruzione di piani di welfare interaziendali che vedono coinvolti l'attore pubblico insieme all'impresa e al sindacato, al fine di realizzare quelle economie di scala che le dimensioni ridotte sfavoriscono. Certo, soprattutto nelle piccole imprese, siamo spesso dentro uno scambio tra incrementi di produttività e moderazione salariale, ancorché mediato dall'accesso a prestazioni integrative di welfare, che riduce il potere delle organizzazioni sindacali (Leonardi e Arlotti, 2012). D'altra parte, tutto questo si svolge in presenza di soluzioni di rappresentanza e tutela da un lato più decentrate, dall'altro anche più segmentate, tra aziende di grandi $o$ piccole dimensioni, tra singole aree territoriali, tra lavoratori occupati stabilmente e lavoratori più ai margini del mercato del lavoro e della gerarchia aziendale (Ascoli, Mirabile e Pavolini, 2013; Ascoli e Agostini, 2014). Di questa grande variabilità occorre tenere conto quando si guarda ai rapporti emergenti tra welfare e relazioni industriali, in special modo in riferimento al tema del welfare contrattuale e aziendale, cui va molta della nostra attenzione. Siamo di fronte a campi che tendono a integrarsi vicendevolmente, all'interno di un quadro soggetto al prodursi di vecchi e nuovi dualismi, e insieme a questi però anche di sostanziali innovazioni nel perimetro dei rapporti tra gli stakeholder territoriali (imprese, istituzioni, parti sociali). Questo in particolare per quello che riguarda la ricerca di incastri istituzionali nuovi o forme di «cooperazione pragmatica» come le hanno definite Burroni e Ramella (2015) per il rilancio della competitività e la produzione di beni collettivi: politiche attive del lavoro, formazione professionale, servizi di conciliazione, a vantaggio delle imprese e dei lavoratori. 
$\mathrm{Su}$ questi fenomeni, le influenze dei contesti istituzionali, locali prima di tutto, hanno un peso determinante. Non meno importante è tuttavia inquadrare questi processi alla luce dei condizionamenti che vengono dalla struttura della domanda di lavoro, per sua larga parte in Italia concentrata in settori a basso tasso di valore aggiunto e di contro con una quota minoritaria di grandi imprese, a più alta produttività e qualificazione del lavoro (si veda da ultimo Reyneri e Pintaldi, 2013). Il riferimento ai condizionamenti delle strutture produttive appare rilevante per almeno due ordini di motivi in relazione al welfare aziendale: da un lato, l'approfondimento di queste dinamiche non può non tenere conto della frammentazione dimensionale e territoriale del tessuto imprenditoriale; dall'altro, questa specificità italiana va analizzata avendo ben presenti le differenze tra piccole e grandi imprese, sia in termini di opportunità che di vincoli al welfare aziendale.

Rispetto all'ampio spettro di soluzioni integrative che rimandano al welfare aziendale nelle pagine che seguono daremo conto dei servizi che più si stanno diffondendo in Italia. Nel fare questo presteremo particolare attenzione alle finalità sottese alle strategie aziendali. La base empirica su cui è stata condotta l'analisi proviene da due indagini svolte da Astraricerche per conto di EdenRed. Una prima indagine - che ha avuto luogo nel maggio 2011 - si è basata su 883 interviste on line condotte su un campione di dipendenti di imprese non operanti nell'agricoltura. La seconda rilevazione è stata invece rivolta alle imprese e si è svolta tra il 10 giugno e il 14 luglio 2011. Sono state interpellate 344 imprese selezionate in modo da rispecchiare la distribuzione nazionale delle aziende per ripartizione territoriale, numero di dipendenti e macrosettore di attività. Anche questa rilevazione è stata condotta attraverso questionario on line (CAWI).

\section{L'incontro tra domanda e offerta di welfare aziendale: alcune evi- denze empiriche}

Le evidenze empiriche che discutiamo in questo lavoro possono essere suddivise in due principali dimensioni. Da un lato il tipo di prestazioni offerte, ovvero il ventaglio e le caratteristiche di fondo dei servizi erogati dalle aziende. Dall'altro le finalità strategiche del welfare aziendale. Questa bipartizione permette di evidenziare anche altre questioni legate a questi dispositivi integrativi, come il rapporto tra domanda e offerta, tra cioè quello che le aziende mettono a disposizione dei propri dipendenti e quello che di contro viene richiesto. Emergono per questa via anche elementi più di livello macro e meso che risultano essere non meno determinanti rispetto alla costruzione di piani di welfare aziendale. Riguardo a questi ultimi è emerso ad esempio quanto la variabile dimensionale e quella territoriale siano aspetti fondanti che determinano specifici sistemi di welfare azien- 
dale, con tutto quello che ne consegue in termini di influenze dei contesti istituzionali locali. Prima di concentrare l'attenzione su questi aspetti è opportuno tuttavia iniziare dal tipo di prestazioni. Da questo punto di vista un primo elemento di riflessione riguarda la varietà dei servizi (non solo peraltro strettamente di welfare) attivi nelle imprese (vedi fig. 1). Dai più diffusi buoni pasto (63\%), alla flessibilità negli orari di lavoro ${ }^{1}$, alla assistenza burocratico-legale e telelavoro, fino a prestazioni più complesse e onerose per le aziende come l'assistenza medica, le convenzioni per gli asili nido, l'assistenza ai non autosufficienti, lo spettro di interventi è assai ampio. Di contro se consideriamo i servizi di welfare in senso lato il panorama che si ha di fronte si restringe. In effetti, ad eccezione dell'assicurazione medica, riscontrabile in $1 / 3$ del campione, la diffusione percentuale dei servizi di cura e assistenza integrativa è scarsa, in particolare per quanto riguarda la tematica della non autosufficienza (solo il 4,5\% del campione). Più alta semmai è l'incidenza delle convenzioni per gli asili nido (12\%) e la dotazione di nidi aziendali (7\%). Considerati congiuntamente coprono il 19,3\% del campione. Si tratta di una percentuale distante rispetto ad altri servizi più diffusi, in ragione soprattutto dei costi elevati per la loro attivazione. Bisogna riconoscere però che il ricorso a questi servizi è tendenzialmente in crescita, come altri studi già hanno confermato (vedi in particolare Pavolini e Carrera, 2013).

Sulla scorta di quanto appena detto è interessante rimarcare una diversità tutta interna a quelli che potremmo definire i servizi aziendali a copertura dei nuovi profili di rischio sociale: invecchiamento della popolazione, non autosufficienza, conciliazione vita-lavoro. La crescita di servizi o facilitazioni in questa direzione sta indubbiamente a testimoniare di un allargamento delle funzioni classiche riconosciute al welfare nelle imprese. Questo allargamento è però circoscritto ai servizi di conciliazione e cura dei minori, lasciando in secondo piano tutto il versante della non autosufficienza. È inoltre principalmente nelle grandi aziende che questi servizi integrativi tendono a emergere di più.

Resta in ogni caso uno scarto considerevole tra domanda e offerta. Ad eccezione dei buoni pasto e della flessibilità nell'orario di lavoro, per il resto dei servizi il gap tra ciò che viene erogato e ciò che viene domandato è significativamente ampio. Basti pensare che i dipendenti che vorrebbero usufruire del telelavoro sono più del $50 \%$ dei rispondenti, mentre ad offrirlo sono meno del $20 \%$ delle aziende interpellate. Per non parlare di asili nido e servizi di cura, compresa la non autosufficienza: a domandarli sono quasi un dipendente su due, mentre a offrirli meno di un datore di lavoro su dieci.

1. Per flessibilità dell'orario di lavoro si intende la possibilità di posticipare o anticipare di un'ora l'entrata o l'uscita. 
Fig. 1 - I servizi offerti dalle imprese, val. \%, anno 2011

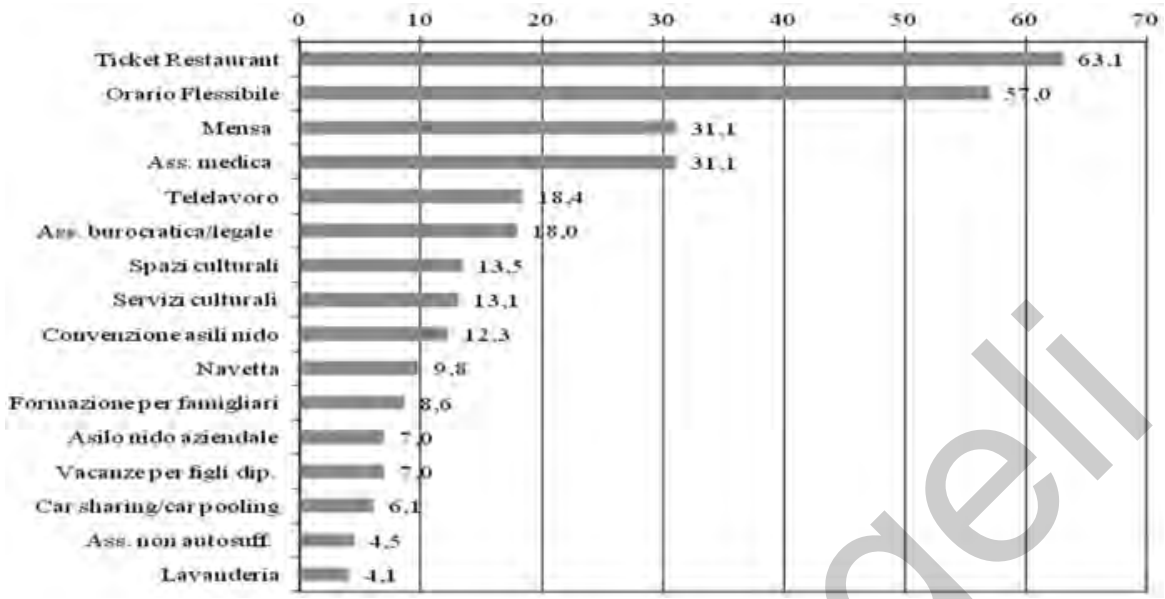

Fonte: nostra elaborazione su dati Astraricerche

A livello regionale la distribuzione dei servizi presenta alcune specificità su cui è interessante focalizzare l'attenzione. Per quanto riguarda la dotazione dei servizi si conferma in tutte le regioni la centralità che sembrano assolvere le prestazioni di conciliazione vita-lavoro, specie nel Centro-Nord e nel Nord-Ovest (fig. 2). Le regioni meridionali sono di contro quelle che hanno una minore dotazione di prestazioni di questo tipo e in generale mostrano un minore sviluppo del welfare aziendale.

Dal punto di vista dei servizi di cura, la performance migliore la registra il Nord-Ovest, che cumula una maggiore dimensione aziendale (in media) e una maggiore occupazione femminile. Le aziende che superano i 250 addetti del Nord Ovest, in particolare le multinazionali dell'industria, hanno sviluppato soprattutto l'assistenza sanitaria, con una particolare rilevanza anche nei confronti della non autosufficienza, attraverso una rete di soggetti convenzionati e grazie a un sistema di broker sul territorio. Diversamente nelle regioni del Nord-Est, soprattutto, vi è una incidenza più ampia dei servizi relativi alla flessibilizzazione dell'orario di lavoro.

Questi dati ci consentono di fare ulteriori considerazioni rispetto alle differenze che corrono tra piccole e grandi imprese ${ }^{2}$. Queste differenze

2. L'analisi che qui presentiamo ha dei limiti determinati dall'accorpamento della classe 16-250 addetti, scelta effettuata in origine da Astraricerche, per cui la nostra lettura non può arrivare a un livello di dettaglio più fine, in particolare per quello che riguarda le medie imprese. Lo stesso possiamo dire del ruolo ma soprattutto delle diverse forme di mediazione istituzionale imputabili alle organizzazioni sindacali. I servizi riportati nel- 
Fig. 2 - I servizi di welfare aziendale offerti dalle imprese indagate. Valori percentuali sul totale delle imprese, val. per ripartizione geografica, anno 2011
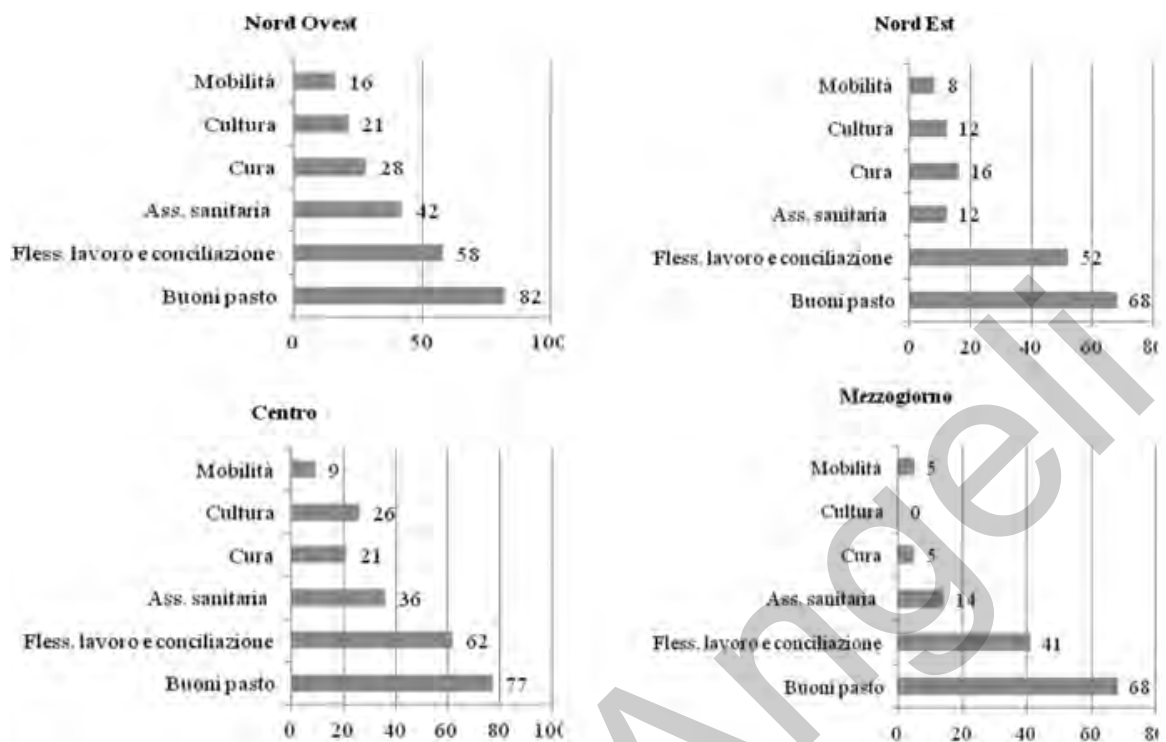

Fonte: nostra elaborazione su dati Astraricerche

sono determinate dall'insieme di vincoli e opportunità che sottostanno allo sviluppo del welfare aziendale, in particolare rispetto al problema delle dimensioni di scala. Le imprese di grandi dimensioni possono mettere in campo più facilmente flexible benefit in proprio, ovvero schemi di retribuzione che integrano il salario del dipendente con servizi per sé e per la sua famiglia, che altrimenti si sarebbero dovuti reperire all'esterno sfruttando le strutture e la numerosità legate proprio alla loro dimensione (Treu, 2013). Viceversa, per i territori a economia diffusa, quelli della tipica piccola e media impresa italiana, i servizi sono più limitati. Emerge ad ogni modo una specificità tipica delle piccole imprese sulla quale vale la pena attirare l'attenzione. Rispetto al panorama complessivo degli interventi sono soprattutto le esigenze legate alla flessibilità negli orari di lavoro e alla conciliazione a riguardare la maggior parte degli accordi aziendali (vedi fig. 3).

la banca dati vengono da accordi contrattati con le parti sociali. Poco ci dicono però delle modalità e delle procedere istituzionali attraverso le quali sono stati prodotti, anche con l'intervento di singole amministrazioni territoriali. 

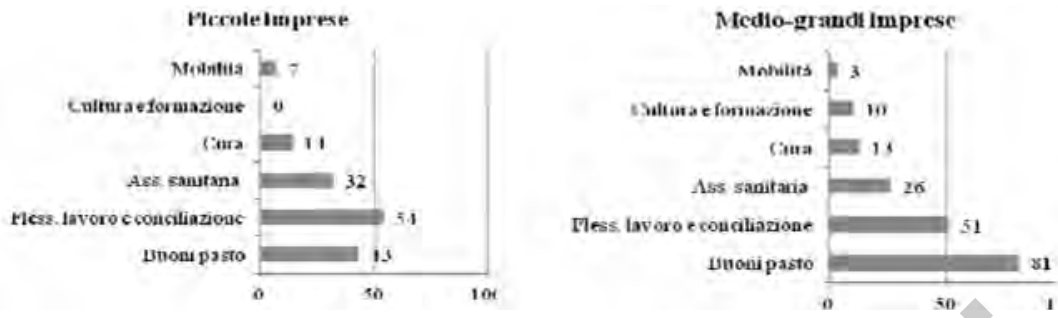

Fonte: nostra elaborazione su dati Astraricerche

Tutto questo se da un lato denota una più ristretta presenza di strumenti di welfare aziendale, dall'altro però li circoscrive ad aspetti connaturati alla struttura della produzione, più esposta ai margini di flessibilità richiesti dai cicli produttivi (vedi anche Pavolini, Mirabile e Ascoli 2013).

\section{Verso una territorializzazione del welfare aziendale?}

L'indagine empirica ha esplorato inoltre quali sono le strategie aziendali sottese all'utilizzo del welfare aziendale nei diversi contesti, sia per raggruppamento territoriale che per dimensione delle imprese. Per quanto riguarda la collocazione territoriale è importante partire da un primo dato di fondo, ovvero la bassa domanda e anche desiderabilità degli strumenti di welfare aziendale nelle regioni del Mezzogiorno (vedi fig. 4). Questa bassa desiderabilità del welfare aziendale nel Mezzogiorno trova probabilmente una prima spiegazione nella bassa dotazione di prestazioni su tutte le gamme di servizi, basilari e più complessi. D'altra parte, la presenza di reti di welfare locale più rarefatte, povere di soluzioni formali, non agevola lo sviluppo dei servizi accreditati, riducendo la portata e le connessioni del welfare aziendale oltre il perimetro dell'impresa. Diversamente, nelle regioni del Centro e del Nord, le percentuali più alte di risposte "positive", si devono non solo a una maggiore densità e presenza dei servizi interni, ma anche alle più fitte relazioni con le reti del welfare locale. 
Fig. 4 - Livello di desiderabilità del welfare aziendale secondo i dipendenti, val. \%, anno 2011

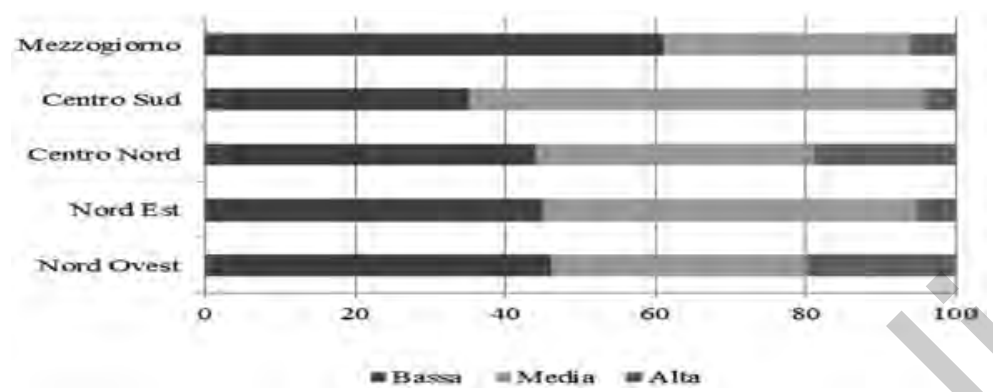

Fonte: nostra elaborazione su dati Astraricerche

Il Mezzogiorno si discosta inoltre in maniera significativa dalle altre ripartizioni territoriali rispetto all'utilità e al senso che le aziende attribuiscono a questi dispositivi (vedi figg. 5 e 6). Indipendentemente dalle dimensioni, le aziende del Sud affermano di considerare il welfare aziendale essenzialmente come mezzo per contrastare la conflittualità interna, diminuire l'assenteismo e altresì garantire benefit alle fasce apicali della gerarchia interna.

Certo anche nelle grandi imprese del Nord-Ovest il welfare aziendale concorre a rimarcare gerarchie interne tra gruppi core apicali e gruppi più periferici all'interno dell'impresa. Qui però siamo in presenza di una desiderabilità molto più alta dal lato dell'utenza e soprattutto a pacchetti di servizi più strutturati che danno conto di un distinto orientamento al welfare aziendale, tendenzialmente gerarchico e però anche relativamente esteso. A questo proposito è interessante sottolineare la differenza con le aziende afferenti al raggruppamento Nord-Est, dove l'erogazione dei benefit è principalmente un mezzo per riconoscere premialità alle risorse umane ritenute strategiche (vedi fig. 7). Questa premialità (che non coincide necessariamente con un orientamento gerarchico) è a ben vedere anche l'elemento caratterizzante del welfare aziendale nelle piccole imprese (vedi fig. 8), soprattutto considerando il tipo di servizi e agevolazioni che tendono a essere messi a disposizione dei dipendenti. Come già detto, non tanto piani strutturati di welfare integrativo, né pacchetti di prestazioni volti a legittimare la gerarchia aziendale, bensì servizi e agevolazioni che hanno l'obiettivo di gestire al meglio (e a costi contenuti) l'allocazione delle risorse umane rispetto all'andamento dei cicli produttivi. 
Fig. 5 - Il punto di vista delle aziende: Welfare aziendale come mezzo per contenere $i$ conflitti, val. \%, anno 2011

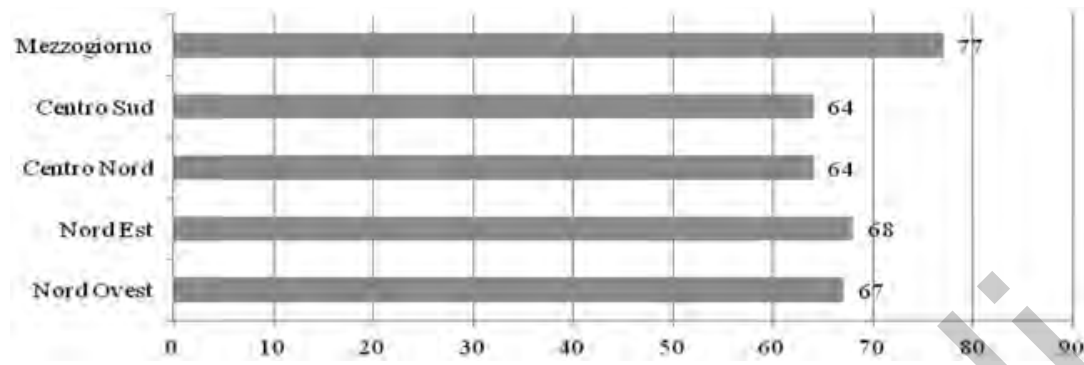

Fonte: nostra elaborazione su dati Astraricerche

Fig. 6 - Il punto di vista delle aziende: Welfare aziendale come premio per fasce apicali di dipendenti, val. \%, anno 2011

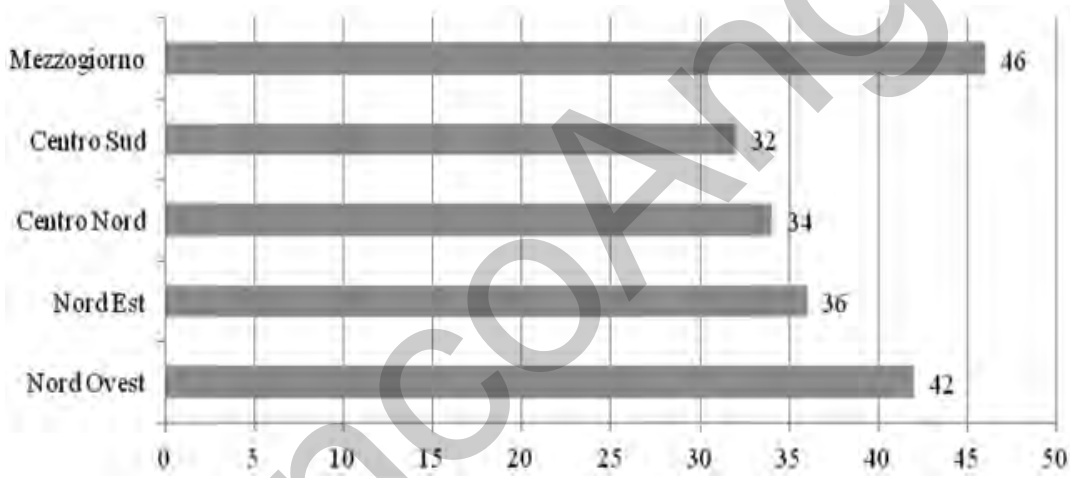

Fonte: nostra elaborazione su dati Astraricerche

Fig. 7 - Il punto di vista delle aziende. Welfare aziendale come strumento per mantenere le risorse umane critiche ritenute indispensabili, val. \%, anno 2011

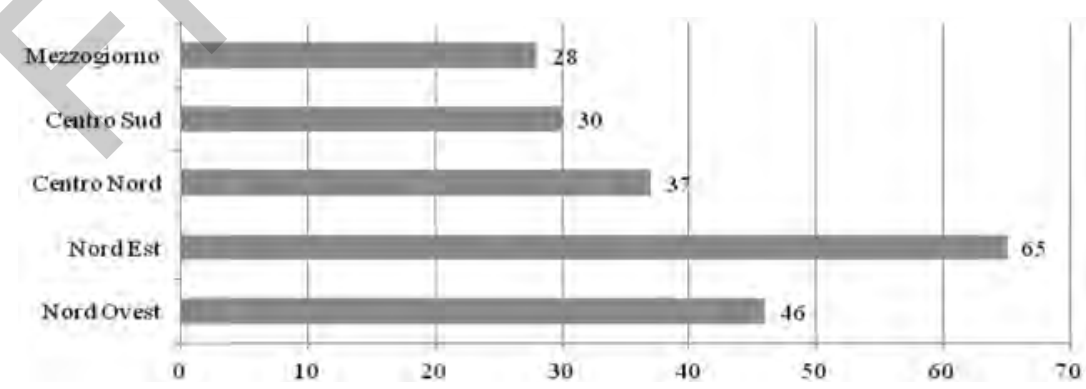

Fonte: nostra elaborazione su dati Astraricerche 
Fig. 8 - Il punto di vista delle aziende. Welfare aziendale come mezzo per trattenere risorse critiche ritenute indispensabili, val. \%, anno 2011

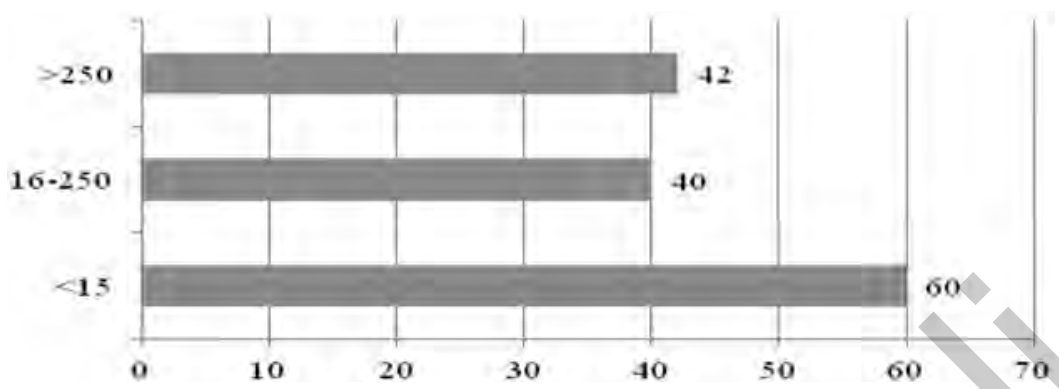

Fonte: nostra elaborazione su dati Astraricerche

\section{Limiti e prospettive del welfare aziendale}

L'analisi che abbiamo condotto ci consente di tornare su alcune delle questioni che sono state poste in avvio di questo lavoro circa le funzioni che gli strumenti di welfare aziendale assolvono rispetto alla grande varietà di istituti contrattati fuori e dentro le aziende. Al pari di altri strumenti promossi dalla contrattazione, ad esempio gli enti bilaterali (Burroni e Carrieri, 2011; Regalia, 2012; Burroni e Pedaci, 2014), anche il welfare aziendale è in crescita, in particolare sul fronte della conciliazione vita-lavoro e della cura dei minori. Dalla possibilità di combinare con più flessibilità gli orari di lavoro, ai nidi aziendali e in convenzione, fino a facilitazioni nel disbrigo di faccende domestiche e amministrative/legali, siamo certamente di fronte a un tentativo di allargare il ventaglio delle prestazioni offerte dalle aziende. Resta una estensione di queste prestazioni non solo molto di sotto della domanda ma anche suscettibile di forti differenziazioni per quel che riguarda la loro dotazione e altresì nei modi di concepirne le funzioni strategiche da parte delle aziende. Siamo d'altra parte a pieno titolo dentro una prospettiva di ripresa delle tendenze dualistiche nel mercato del lavoro che ha effetti diretti sulle modalità di integrazione tra welfare e relazioni industriali. Tanto più in un paese come l'Italia, storicamente attraversato da molteplici fratture su base categoriale e professionale, tra aree geografiche, tra lavoratori più garantiti e outsider. Pur tuttavia è in questo quadro che i vari i dispositivi contrattuali e aziendali tendono oggi a integrare, in alcuni casi colmare, gli spazi vuoti lasciati dall'intervento pubblico, pur al netto dei dualismi che essi stessi determinano.

Il primo dei dualismi che emerge dall'analisi riguarda il sistema di servizi nelle grandi e nelle piccole imprese. Se nelle prime, in particola- 
re quelle localizzate nel Nord-Ovest, il welfare aziendale tende a comporsi di un ventaglio relativamente più ampio di interventi, nelle piccole esso assume connotazioni strutturalmente diverse. In questo caso il sistema che emerge si connota per la prevalenza di forme integrative di premialità che appaiono strumentali prima di tutto alla gestione dei cicli (e dei picchi) produttivi (da qui l'alta incidenza delle agevolazioni sull'orario di lavoro) e al conseguente mantenimento delle risorse umane più strategiche. Non appare certo un caso che questo emerga in particolare per le regioni del Nord-Est, in cui più forte e radicata è la presenza di sistemi di piccola impresa, tradizionalmente esposti a forti picchi di flessibilità nella gestione dei cicli produttivi.

In questa stretta sinergia tra welfare aziendale e ciclo produttivo, le componenti della domanda di lavoro non sono estranee. Certo l'impulso dato dalla legislazione è un fattore che incide sulla profittabilità o meno di certi dispositivi aziendali. Pur tuttavia la loro configurazione e soprattutto le funzioni che gli vengono demandate non agiscono indipendentemente dai condizionamenti riconducibili alle strutture produttive, così come alle influenze dei contesti istituzionali, in particolare locali. C'è semmai un rapporto di reciproco rimando tra queste determinanti, con effetti a nostro parere decisivi rispetto alla scelta dei servizi e delle agevolazioni introdotte. $\grave{E}$ in un quadro di questo genere che va colto del resto quello scambio tra produttività e moderazione salariale, mediata però da servizi integrativi, che tende a riguardare soprattutto i territori a economia diffusa. Recuperi di produttività, flessibilità nell'organizzazione e nei tempi di lavoro ma anche moderazione salariale e premialità nei confronti delle risorse umane strategiche, fanno da cornice al tipo di welfare aziendale che tende a emergere nei sistemi di piccola impresa.

Molto diverso è il sistema di welfare aziendale delle grandi imprese. In primo luogo per via di pacchetti di servizi più strutturati. E questo era lecito aspettarsi. In secondo luogo per la presenza, già in partenza, di economie di scala che solo le grandi dimensioni sembrano consentire di raggiungere quando si tratta di garantire condizioni di vantaggio, per esempio nella stipula di assicurazioni sanitarie, o servizi integrativi per il tramite di broker o agenzie specializzate esterne. Il dato più interessante è comunque un altro. E cioè il fatto che questa più ampia dotazione di prestazioni è mediata da un orientamento gerarchico, finalizzato soprattutto a riconoscere differenze tra dipendenti apicali, a cui vanno i maggiori vantaggi delle provvidenze integrative, e gruppi collocati più in basso nella organizzazione aziendale. Da questo punto di vista se le piccole imprese sfruttano il welfare aziendale secondo un approccio premiale e strumentale, in larga parte tarato sulla gestione dei cicli produttivi, nelle grandi emerge un orientamento più strettamente gerarchico, premiale semmai delle fasce alte della gerarchia aziendale. 
Questo tratto gerarchico, ma con sue proprie specificità, ha una attinenza rispetto ad una ulteriore differenziazione territoriale che riguarda nello specifico le regioni meridionali. Il welfare aziendale nelle regioni del Sud è molto meno presente e anche meno domandato. La differenza nella dotazione di servizi di welfare esterni alle imprese è un fattore determinante nella connessione tra impresa e territorio. E qui si misura una forte differenza tra Nord e Sud, da ricondurre al diverso peso del capacity building proprio delle istituzioni locali nel loro ruolo di facilitatori e produttori di servizi, sia pure in partnership con i provider privati.

Stante così le cose, la prospettiva di un aumento delle disuguaglianze territoriali tra Nord e Sud è un effetto tangibile dei processi che vanno interessando il welfare aziendale e a ben vedere anche quello contrattato al di fuori delle aziende. D'altra parte la possibile diffusione di incastri distorti o strumentali tra welfare pubblico e welfare aziendale puô incrementare questa distanza, tutta a sfavore dei territori più deboli, con il rischio di cumulare nei fatti due svantaggi: il primo derivato dalla scarsa presenza del welfare esterno alle aziende, pubblico o pubblico-privato, il secondo da meccanismi isomorfici che riproducono modelli strumentali o particolaristici. Va detto infatti che non necessariamente le logiche di imitazione tra aziende inducono effetti positivi. Al contrario ci possono anche essere effetti negativi, ad esempio una diffusione di pratiche strumentali, alla stregua di privilegi ad personam o slegati da logiche di incentivazione e riconoscimento della produttività.

Sulla base di quanto detto ci possiamo domandare quali siano le variabili indipendenti che incidono sulla diffusione del welfare aziendale. Una variabile determinante, legata alla struttura produttiva, appare senza dubbio la dimensione aziendale. Tra grandi e piccole imprese sono diversi non solo i pacchetti di servizi ma più in generale le condizioni e gli orientamenti, così come le procedure attraverso cui si provvede alla costruzione di questi interventi. Accanto a questo, tuttavia, non meno decisiva appare la collocazione territoriale. A ben guardare sono infatti tre i profili di welfare aziendale che emergono dalla nostra analisi: quello tipico dei territori a economia diffusa, votato alla flessibilità e ad agevolazioni sugli orari di lavoro, visti però come mezzo attraverso cui incidere in primis (se non soprattutto) sulla produttività del lavoro, in particolare delle risorse umane più strategiche ai fini dei cicli produttivi; quello della grande impresa del Nord-Ovest, di cui abbiamo già detto e che qui possiamo sintetizzare in un modello più dotato di prestazioni da un lato, ma anche più gerarchico e corporativo dall'altro; infine il welfare aziendale del Mezzogiorno. Nella misura in cui è presente, quest'ultimo tipo si caratterizza per un sorta di combinato disposto tra esigenze legate alla gestione della conflittualità interna e una distribuzione gerarchica e particolaristica dei benefit a vantaggio del vertice aziendale. 
Rimane in parte inevasa, come già rimarcato, una questione su cui ulteriori approfondimenti futuri potranno offrire spunti interessanti, ovvero quanto ma soprattutto come il welfare aziendale è mediato dalle parti sociali. E in che misura queste pratiche siano non solo esportabili ma anche generalizzabili, nel senso di avere una rappresentatività superiore a quella ipotizzabile sulla base di singoli studi di caso - territoriali o aziendali - cui fino ad oggi è andata molta dell'attenzione degli studi condotti sul tema.

\section{Riferimenti bibliografici}

Agostini C., Ascoli U. (2014). Il welfare occupazionale: un'occasione per la ricalibratura del modello italiano? Politiche Sociali, 2: 263-279. DOI: $10.7389 / 77343$

Burroni L., Carrieri M. (2011). Bargaining for social rights, (BARSORI) country report Italy, testo disponibile al sito: www.uva-aias.net/uploaded_files/regular/ BarsoriReport-Italy.pdf (11-11-2014).

Burroni L., Pedaci M. (2014). Collective Bargaining, Atypical Employment and Welfare Provisions: The Case of Temporary Agency Work in Italy. Stato e Mercato, 2: 169-194 DOI: 10.1425/77410

Burroni L., Ramella F. (2015). Negoziare, Regolare e Promuovere lo Sviluppo Locale. Rivista Giuridica del Lavoro, 1: 49-63.

Carrieri M., Treu T., a cura di (2013). Verso nuove relazioni industriali. Bologna: il Mulino.

Ferrera M., Maino F. (2013). Primo Rapporto Sul Secondo Welfare In Italia, Percorsi di secondo welfare, testo disponibile al sito: www.secondowelfare. it/edt/file/PRIMO_RAPPORTO_SUL_SECONDO_WELFARE_IN_ITALIA. pdf (11-11-2014).

Gori C. (2012). L'alternativa al pubblico? Milano: FrancoAngeli

Leonardi S., Arlotti M. (2012). Welfare contrattuale e bilateralismo. La Rivista delle Politiche Sociali, 3: 77-114.

Mallone G. (2013). Il secondo welfare in Italia: esperienze di welfare aziendale a confronto, Working paper, Percorsi di secondo welfare - Centro Einaudi, Torino, testo disponibile al sito: www.secondowelfare.it/working-paper/ esperienze-di-welfare-aziendale-a-confronto.html (11-11-2014).

Pavolini E., Carrera F. (2013). I tratti del welfare occupazionale a partire dalle indagini quantitative. In: Pavolini E., Mirabile M.L., Ascoli U., a cura di, Tempi moderni. Il welfare nelle aziende in Italia. Bologna: il Mulino.

Pavolini E., Mirabile M.L., Ascoli U., a cura di (2013). Tempi moderni. Il welfare nelle aziende in Italia. Bologna: il Mulino.

Regalia I. (2012). La protezione sociale del lavoro come tema delle relazioni industriali. La Rivista delle Politiche Sociali, 3: 13-36.

Reyneri E., Pintaldi L. (2013). Dieci domande su un mercato del lavoro. Bologna: il Mulino.

Treu T. (2013). Welfare aziendale. Roma: Ipsoa. 